\title{
Non-destructive monitoring of tensile of mild steel samples by magnetic Barkhausen and ultrasonic methods
}

\author{
Yonka Ivanova ${ }^{1,2, *}$ \\ ${ }^{1}$ Sofia University “St.Kl.Ohridski”, Faculty of Physics, Sofia, Bulgaria \\ ${ }^{2}$ Institute of Mechanics-BAS, Solid Mechanics, Sofia, Bulgaria
}

\begin{abstract}
The paper presents an experimental study of deformations of mild steel samples by magnetic Barkhausen, magnetoacoustic and ultrasonic methods. The purpose of the study is to investigate and analyze the dependence between mechanical, magnetic and acoustic properties and to find appropriate parameters for stress state evaluation in the elastic and plastic regions.
\end{abstract}

\section{Introduction}

The working conditions of the materials lead to degradation of microstructure and properties of engineering materials. Among mechanical properties, static tensile properties are fundamental and have been used as a standard to represent mechanical properties. When materials deform, elastic deformation occurs in an early stage. When the deformation exceeds one critical value, named yield point, the deformation becomes plastic. The increasing of the mechanical stress beyond the yield stress has an undesirable effect because of subsequent plastic deformation and unpredictable failure. In such a manner, tensile properties have been crucial for discussion of plastic deformation process. The destructive methods such as tensile, compression or bending are traditionally used for obtaining the mechanical properties. Non-destructive techniques offer an alternative to traditional methods.

Non-destructive testing (NDT) methods enable easy, fast and "in situ" receiving the information for materials on the base on the correlations between NDT parameters and mechanical characteristics. Thus the researchers can study the alteration of microstructure and physical properties during exploitation (stress, deformation, heat treatments, neutron irradiation etc.). Many research papers concerning the stress effect on non-destructive testing parameters reveal results due to different materials and microstructure as well as NDT parameters $[1-10,11,12,15,16,33,34]$. The most used methods for stress (residual and applied stress) and structure evaluation are magnetic [1, 2, 15, 16, 22, 24, 35], magnetic Barkhausen Noise (MBN) [18-25], magneto acoustic emission (acoustic Barkhausen noise) (MAE) $[9,11,12,13,14]$ and ultrasonic methods $[4,5,26-29,30,31]$. The first two

\footnotetext{
* Corresponding author: yonka@imbm.bas.bg
} 
methods and effects are due to abrupt movement of magnetic domain walls during the magnetization of samples. They are sensible to microstructure (carbon content, grain size etc., chemical composition) and stress state of materials [10,12, 14, 17, 21]. The working principle of MBN is based on Barkhausen noise when a ferromagnetic material is subjected to a varying magnetic field $[14,21]$. The MBN parameters depend on penetration depth of magnetic field, frequency and physical properties such as specific electrical conductivity and magnetic permeability. The detected MBN signal is obtained from depths less than 0.2 $\mathrm{mm}$ below the surface, i.e. MBN is a surface technique [9, 11-13]. MAE is associated with the generation of acoustic signals as a result of continuous changes in magnetization and change of magnetostriction in separate areas of the material. The MAE parameters are obtained from the whole volume of material [8, 9, 11-13]. The third method is ultrasonic. It uses the variation of ultrasonic wave velocity as a function of stress level on the base of the acoustic-elastic theory. Internal stress measurement can be classified into groups according to the different type ultrasonic waves (longitudinal, transverse, longitudinally refracted and surface waves) [3-5, 26-30]. The choice of NDT information parameters is difficult because of complexity of many factors such as microstructure features and stresses. The complicated problem has a solution by applying several NDT methods based on different physical approaches [1-7].

The aim of the study is to perform monitoring of the deformation during uniaxial tension by three NDT methods. Comparing the results from surface techniques such as magnetic Barkhausen noise and ultrasonic surface wave methods with volumetric one magneto-acoustic emission gives the possibility to find out the appropriate non-destructive parameter for evaluation of applied stress. The combination of NDT methods for stress state evaluation in elastic and plastic regions the stress-strain diagram allows obtaining more information about materials and it is still topical. The variation of the Rayleigh wave velocity in the elastic and plastic regions of the stress-strain diagram of steels has to be obtained experimentally and compared with the results from another surface method. Another research goal is to establish the sensitivity and reliability of used methods to plastic deformation and damaging process of materials.

\section{Experimental methodologies}

Experimental procedure for investigation of deformation during tensile in low carbon steel specimens includes the following stages:

- Preparation of specimens;

- Tensile testing;

- MBN and MAE measurements during tensile testing.

- Ultrasonic surface waves measurements, carried out on-line during tensile testing

\section{Materials and tensile testing}

The specimens are obtained from a $3 \mathrm{~mm}$ thick hot rolled mild steel plate. The dimensions of working part of specimens are $160 \mathrm{~mm}(\mathrm{~L}) \times 30 \mathrm{~mm}(\mathrm{~W}) \times 3 \mathrm{~mm}(\mathrm{~T})$ so that the specimen axis was parallel to the rolling direction.

Tensile tests are performed using a universal tensile machine, equipped with a load cell of $100 \mathrm{kN}$. The mechanical properties (yield strength, ultimate tensile strength and elongation after fracture) are preliminary determined from a stress-strain diagram after tension tests. The speed of the loading is $0,5 \mathrm{~mm} / \mathrm{s}$. The yield stress is about $300 \mathrm{MPa}$ and tensile strength is about $410 \mathrm{MPa}$. 


\section{NDT measurements}

The experimental system for in-line non-destructive monitoring is illustrated in the Fig.1. It consisted of tensile testing machine and NDT apparatus for MBN, MAE and ultrasonic surface wave setups. The NDT measurements are completed before reaching the maximum loading force and forming necks in the specimens. No measurements have been made after unloading.

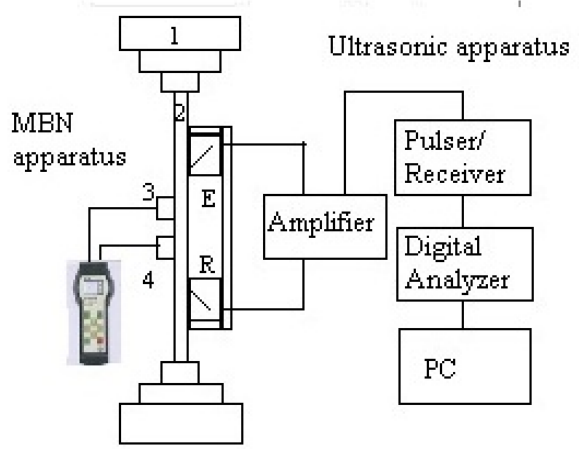

Fig.1.Experimental system for real time NDT monitoring using MBN, MAE (MULTITEST 010) and ultrasound. 1-Tensile machine, 2-tensile specimen, 3-MBN probe, 4-MAE probe

\subsection{MBN and MAE measurement}

The MBN apparatus consists of device 'MULTITEST MC010', contact magnetic-noise probe and acoustic emission probe type $[5,32]$. The principle of operation and methods for calibration of "MULTITEST-MC10" are presented in [32]. The device consists of signal generator, amplifiers of the magnetic and acoustic noise, microprocessor system and programmable software, for controlling the frequency and the magnitude of magnetic excitation. The excitation is by sine wave signal generator. The excitation frequency is set to $80 \mathrm{~Hz}$. The MBN probe contains a magnetic exciter ("U" ferrite core magnet) and MBN pick-up sensor situated between the magnetic poles. The magnetic head was perpendicular to the surface of the specimens. The MBN is acquired using adjustable filters up to 100 $\mathrm{kHz}$. To detect MAE signals an AE sensor (150 kHz, VS $150 \mathrm{MVallen}$ system) with a preamplifier included $(40 \mathrm{~dB})$ was positioned and fixed on the bottom surface of the specimens by specially made holder. The acoustic emission sensor was coupled to the surface of the specimens using silicon acoustic couplant that insures good surface contact between the two surfaces of the samples and facilitates the transmitting of acoustic waves.

The informative non-destructive parameters are Root Mean Square (RMS) values of magnetic noise voltage $\left(U_{m}\right)$ and RMS values of magneto acoustic voltage $\left(U_{a}\right)$. They are measured during the increase of the magnetizing current from 0 to $500 \mathrm{~mA}$, saved in the device memory for signal processing and graphically presented. The processing and analysis is developed in a MATLAB environment. The applied magnetic field is perpendicular to the tensile stress direction. The parameters of magnetization and signal analysis are tunable in order to select the optimal ones as well as to obtain the maximum information of surface structure and stress state. 


\subsection{Ultrasonic measurement}

When ultrasonic Rayleigh wave propagates in the same direction as the applied uniaxial stress, the dependence of the velocity on the tensile stress $(\sigma)$ can be described in the form [26-28].

$$
d C_{R}=\frac{C_{R x}-C_{R o}}{C_{R o}}=\beta_{R} \cdot \sigma
$$

The relative change of surface wave velocity is proportional to the change in time of flight $\left(d t_{R}\right)$ of the wave propagating along a distance L.

$$
\begin{aligned}
& d C_{R}=-d t_{R} \\
& d t_{R}=\frac{t_{R}-t_{R o}}{t_{R o}}=K . \sigma
\end{aligned}
$$

where $t_{R o}$ is the time of flight of wave passing the distance L in an sample without stress, $t_{R}$ denotes the time of flight during the applied stress in the same direction as the load, $K$ is an acoustoelastic coefficient.

The setup for real time ultrasonic measurement is an ultrasonic US box, consisted of pulser and receiver, a $160 \mathrm{MHz}$ A/D converter with 12-bit resolution and a notebook. For our investigation we choose surface Rayleigh ultrasonic waves. They were excited by an angular transducer with variable angle at $4 \mathrm{MHz}$. The propagation depth of these waves are of the order of $1.5 \lambda_{R}$ (Rayleigh wave length) and are about $1.5 \mathrm{~mm}$. The signal was emitted by transducer E, received by transducer $\mathrm{R}$ and recorded as shown in Fig.3. Two transducers were mounted on the specimens in a pitch-catch arrangement by guiding device that allowed the movement of the receiver and ensured the precision of time of flight measurements. The measurements were carried out under same conditions. The time between loading steps is sufficient for recording the signals. The ultrasonic signals obtained from the stressed surface under increasing loading are stored in the computer for further signal processing and compared to reference signal from the stress-free sample.

During tensile testing, the ultrasonic measurements were carried out without stopping the tensile machine to ultimate stress strength. The distance between the transmitter and receiver was kept by the holder and does not change. The propagation of the wave is parallel to the stress direction. The signals corresponding to different loads were saved. The arrival times of flight are calculated by zero crossing technique. The measuring procedures were repeated in order to diminish problems of coupling or thermal changes. For analysis was used the relative difference in time of flight $d t_{R}$, defined as (3).

\section{Results and discussion}

\subsection{MBN and MAE measurements}

In order to carry out MBN analysis the test specimens were loaded with gradually increasing tensile force and after small retention the magnetic characteristics RMS values of MBN voltage $\left(U_{m}\right)$ and MAE voltage $\left(U_{a}\right)$ were measured and were used to characterize the microstructural and stress state of the materials. 
Figure 2 presents calibration curves: RMS values of magnetic noise voltage $\left(U_{m}\right)$ and magnetic acoustic emission $\left(\mathrm{U}_{\mathrm{a}}\right)$ on the excitation current $\left(\mathrm{I}_{\mathrm{n}}\right)$ and their dependences on loading tensile forces $\mathrm{P}$. The magnetizing probe was placed in the middle of specimens, so the magnetic field was perpendicular to stress direction. The acoustic emission transducer was fixed on the other side of the specimens at the same position in all the measurements.

At the zero tensile stress, the MAE parameter is seen to be highest. With increased the loading to and after yield point, MAE parameter reduces values.

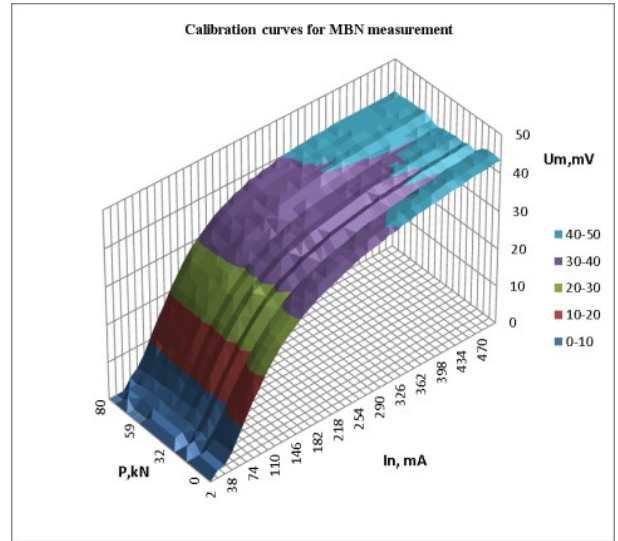

$\mathrm{a}$

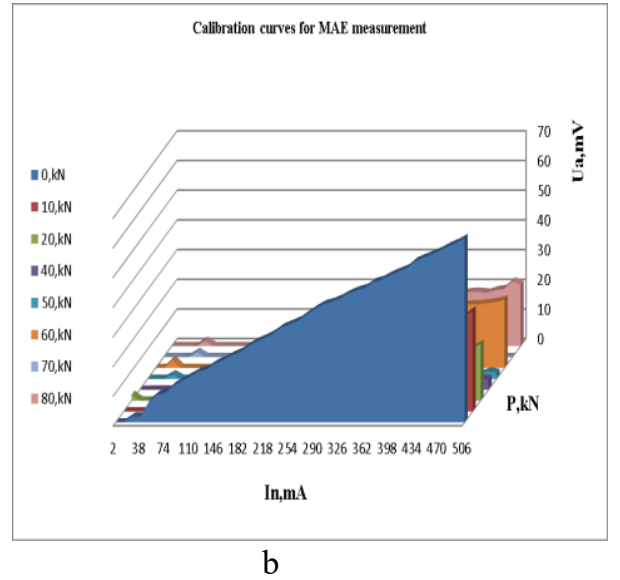

b

Fig.2. Calibration curves for MBN (a) and MAE measurement (b)

The stress-strain diagram and the variation of RMS value of $M B N\left(U_{m}\right)$ are presented in Fig.3a. MBN signal is quite sensitive to stress during the elastic stage, and has a good linear relationship with stress in the plastic stage.

Figure $3 \mathrm{~b}$ show the behaviors of MBN and MAE parameters with applied tension stress $(\sigma)$. It can be seen from the curves: from 0 to $100 \mathrm{MPa}, \mathrm{U}_{\mathrm{m}}$ rises with the stress. As the stress continues to increase from about $100 \mathrm{MPa}$ to the yield stress $\left(\sigma_{y}\right)$, the magnetic parameters start to decrease slowly. Upon the reach of yield limit the RMS value of MBN abruptly drops. The rising of the strain causes small growth of Um. At the beginning of plastic region $\mathrm{U}_{\mathrm{m}}$ slowly increases.

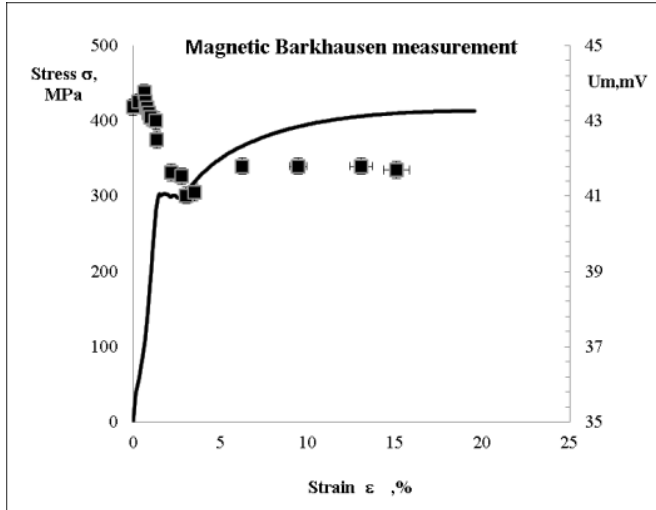

Fig.3a. Stress-strain curve and $\mathrm{MBN}$

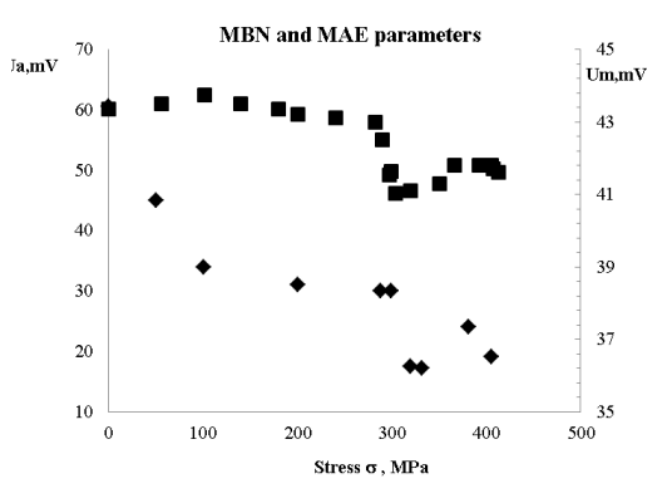

Fig.3b.Variations of RMS values of $\mathrm{MBN}$ and MAE parameters vs. tensile stresses 
In the elastic region the behavior of MBN can be explained with changes of domain configuration as a response of variation of interatomic spacing. The domains within individual grains must reorient to minimize their energy. Stefanita and others [18,25] proposed two mechanisms for explanation of domain pattern. The first one is an orientation of $180^{\circ}$ domains into the direction closest to the stress direction. The second mechanism involves the rising of domain wall numbers. The tensile stress creates more domain walls in the stress direction. Hence in this stage, the MBN signal rises with the increase of the applied stress $[10,14]$. Further increase of the stress causes the augmentation of the dislocation density. In the material, the regions with high dislocation density are separated by regions with low dislocation density, and then dislocation tangles start to form. The newly emerging dislocation tangles and cell boundaries increases the number of microscopic pinning sites. They act as obstacles to domain walls movement $[14,18,25]$. With alteration within the deformation of the microstructure MBN parameter decreases with the increasing of the stress. The theoretical analysis discussed above is in agreement with the experimental results.

Figure 4a,b show the variations of dimensionless parameters $d U_{m}$ and $d U_{a}$ versus parameter $\sigma / \sigma_{y}$. The relative change of MBN $d U_{m}$ and MAE $d U_{a}$ can be defined by:

$$
\begin{aligned}
& d U_{m}=\frac{U_{m}-U_{m o}}{U_{m_{o}}} \\
& d U_{a}=\frac{U_{a}-U_{a o}}{U_{a_{o}}}
\end{aligned}
$$

Where $U_{m}$ and $U_{a}$ are the values of magnetic noise and MAE corresponding to current stress; $U_{m o}$ and $U_{a}$ are the values of magnetic parameters corresponding to unstressed sample.

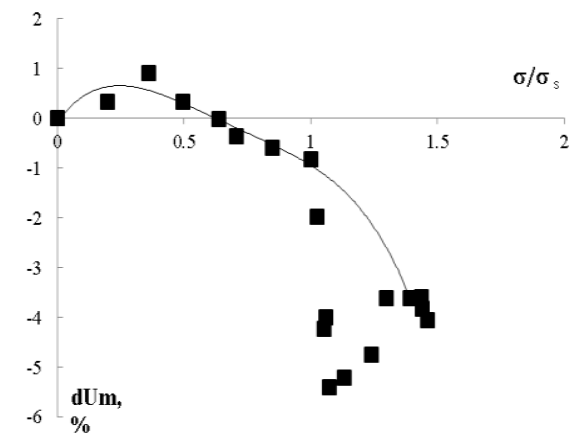

a

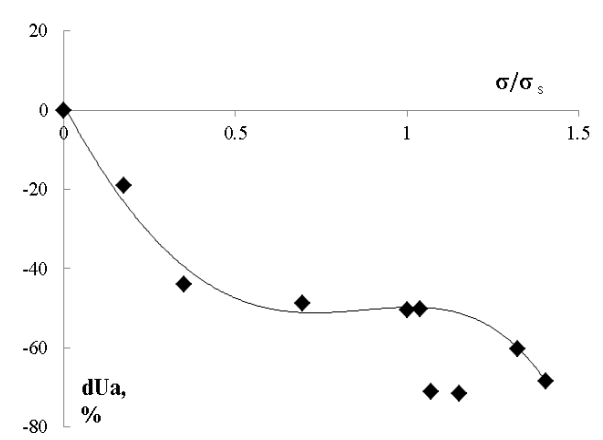

b

Fig.4. Variations of relative changes of parameters dUm (a) and dUa (b) vs normalized stress

With increasing load the material passes from elastic to plastic state and magnetic BN and MAE parameters decrease. The results show that the MBN and MAE parameters are sensitive to the initial stage of plastic deformation, especially for the stresses close to the yield stress. 


\subsection{Ultrasonic measurement}

Figure 5 show the stress-strain curve and variation of ultrasonic parameter $d t_{R}$ during the tensile deformation. The strain and $d t_{R}$ are presented on x-axe. The values $d t_{R}$ are obtained from repeated experiments and closely distributed. It can be observed that the ultrasonic parameter grows with increasing of applied stress. In the elastic region the stress is linearly proportional to the strain and the ultrasonic parameter varies almost linearly with tensile stress.

The dependence between the time of flight $d t_{R}$ and stress in elastic region is expressed by linear regression:

$$
d t_{R}=K_{1} \cdot \sigma
$$

where $K_{1}$ is a coefficient of proportionality between stress and ultrasonic parameter.

In the plastic region the variation of ultrasonic parameter can also be approximated by power low equation:

$$
d t_{R}=K_{2} \cdot\left(\sigma_{\text {plastic }}\right)^{p}
$$

Using least square fitting of the data, the coefficients are obtained: $K_{1}=1,0845.10^{-5} \mathrm{MPa}$, $K_{2}=2,10^{-18} \mathrm{MPa}, \mathrm{p}=6,1$.

The presentation of the results in relative units allows comparing results obtained for different type of materials. Figure 6 presents the variation of dimensionless parameter $d t^{*}\left(d t^{*}=d t_{R(\sigma)} / d t_{R\left(\sigma=\sigma_{y}\right)}\right)$ upon the normalized stress $\sigma^{*}\left(\sigma^{*}=\sigma / \sigma_{y}\right)$.

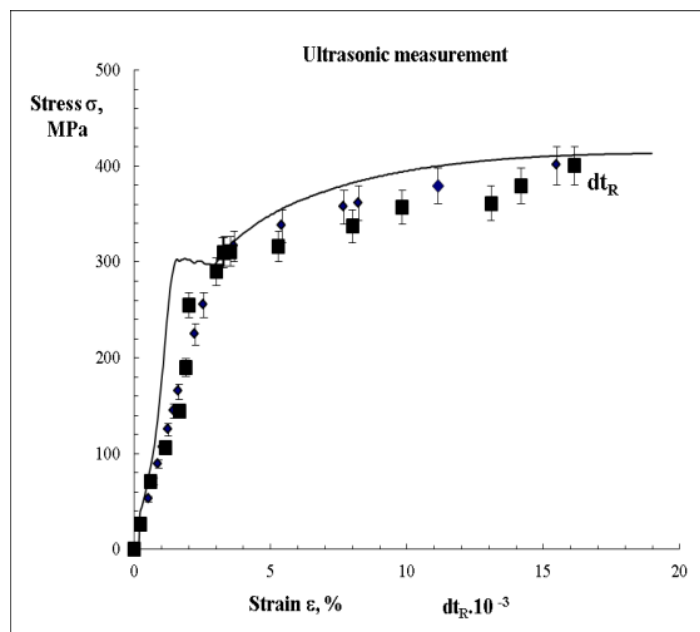

Fig.5. Stress-strain curve and variation of ultrasonic parameter $d t_{R}$ during tensile

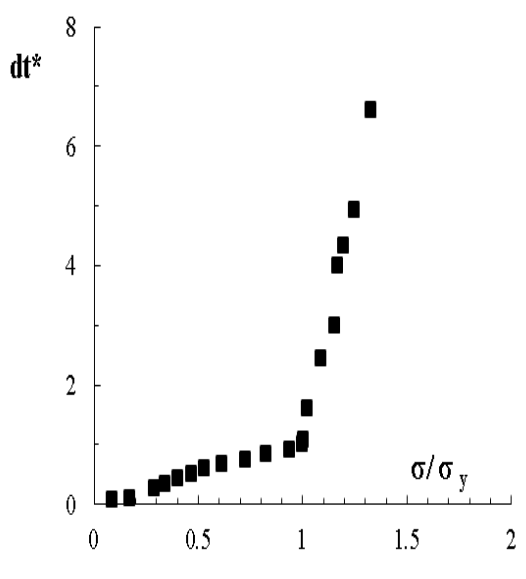

Fig.6. Variation of normalized ultrasonic parameter $d t^{*}$ vs. normalized stress 
The dimensionless parameter $d t^{*}$ is time of flight of ultrasonic Rayleigh waves referred to its value in the end of elastic region and the normalized stress $\sigma^{*}$ is the current value of stress referred to the yield stress $\sigma_{y}$. In the plastic region the parameter $d t^{*}$ sharply increases, i.e. the relative change of ultrasonic Rayleigh waves abruptly decreases.

\section{Conclusion}

The obtained results characterize the variations of NDT parameters: magnetic, magnetoacoustic and ultrasonic (velocities of ultrasonic Rayleigh waves) parameters in low carbon steels samples during tensile stress. They are in good agreement with the theory and experiments and can be explained by deformation processes in the elastic and plastic areas. Due to the relationship with applied tensile stress at different stages, the combination of the three techniques offers the potential for developing the methodology for the study of elastic-plastic behavior of ferromagnetic materials. The sharp change in non-destructive parameters at mechanical stresses close to the yield stress can be used as an indicator of plastic deformation.

\section{References}

1. E. Schneider, I. Altpeter, W.Theiner, Nondestructive methods for material property determination (Boston, Springer, 1984)

2. P. Holler, Nondestructive characterization of materials (New York: Springer, 1987)

3. C.H. Gur, I. Cam, Mater Charact. 58, 5, 447-454 (2007).

4. P. Pereira, F. Armando, F. Grijalba, A Santos, J.Strain Analysis 51, 8, 563-571(2016)

5. Y. Ivanova, T. Partalin, Russ J Nondestruct Test 48, 2, 137-146 (2012)

6. I. Tomas, G. Vertesy, S. Barroso, S. Kobayashi, Nucl Eng Des. 265, 201-209 (2013)

7. S. Takahashi, S. Kobayashi, I. Tomáš, L. Dupre, G. Vértesy, NDT and E Int. (to be published)

8. D.J. Buttle, C. B. Scruby, G.A.Briggs, J.P.Jakubovics, Proc $R$ Soc Lond A 414, 1847, 469-497 (1987)

9. M.Shibata, K.Ono, NDT\&E Int. 14, 5, 227-234 (1981)

10. J.L.Rodriguez, J.A. Perez-Benitez, J. Capo-Sanchez, Rev Mex Fis Journal S 54, 2, 5153 (2008).

11. V.N. Kostin, D.Y. Filatenkov, O.N. Vasilenko, Al.N. Stashkov, 11 ECNDT, Prague 2014 (2014).

12. V. N. Kostin, O.N.Vasilenko, D.Y. Filatenkov, Y.A. Chekasina, E.D. Serbin, Russ J. Nondestruct. Test. 51, 10, 624-632 (2015)

13. V. N. Kostin, D.Y.Filatenkov, Y.A. Chekasina, O.N.Vasilenko, E.D.Serbin, Acoust. Phys. 63, 2, 237-244 (2017)

14. E. S. Gorkunov, Yu. N. Dragoshanskii, V. A. Khamitov, Russ J Nondestruct Test. 37, 12, 835-858 (2001)

15. E.S.Gorkunov, Yu.V. Subachev, A.M. Povolotskaya, S.M. Zadvorkin, Russ J Nondestruct Test. 49, 10, 584-594 (2013).

16. E.S.Gorkunov, A.M. Povolotskaya, S.M. Zadvorkin, E.A. Putilova, AIP Conf. Proc, 1785 (2016)

17. D. M. Stewart, K.J. Stevens, A.B. Kaiser, Curr. Appl. Phys. 4, 308-311 (2004)

18. A. Dhar, L.Clapham, D.L.Atherton, NDT \&E Int. 34, 507-514 (2001)

19. J. Pala, J. Bydžovský, AEEE 7 (2008)

20. T.Ren, G. Chen, W. Zhang, Z Qiu, Appl Mech Mater. 455 (2014)

21. Y. Huijuan and all, Telkomnika 12, 3, 623-630 (2014) 
22. J. Anglada-Rivera, L.R. Padovese, J. Capo-Sanchez, J Magn Magn Mater. 231, 2, 299306 (2001)

23. D. G. Hwang, H. C. Kim, J. Phys. D, Appl. Phys. 21, 1807-1813 (1988)

24. H. Kikuchi and all, J Magn. 16, 4, 427-430 (2011)

25. C. G. Stefanita, D. L. Atherton, L. Clapham, Acta Mater. 48, 3545-3551 (2000)

26. A. N. Guz, F. G. Makhort, Int. Appl. Mech. 36 , 9 , 1119-1149 (2000)

27. V. V. Klyuev, V. N. Filinov, V. I. Matveev, Nondestructive Testing. A Handbook. Ultrasonic Methods of Testing, Vol. 4, (Mashinostroenie, Moscow, 2007)

28. A. A.Chernoochenko, F.G. Makhort, O.I. Gushcha, Int. Appl. Mech., 27, 38-42 (1991)

29. E. Hu, Y. He, Y.Chen, Appl. Acoust. 70, 2, 356-360 (2009)

30. M. Kobayashi, Int J Plasticity 26, 107-125 (2010)

31. M. Kobayashi, Int J Solids Struct. 121 (2017)

32. G.Velev, Y. Ivanova, A. Markovski, B. Velev, Emerging Technologies in NonDestructive Testing $V$ (Taylor \& Francis Group, London, 499-502, 2012)

33. L. Lyubimova, A.Tashlykov, R.Tabakaev, A.Levin, A.Popov, Eng. Fail. Anal. 81, 45$56(2017)$

34. A.Belyaev, A. Semenov, V. Polyanskiy, D.Tretyakov, Y.Yakovlev, Procedia Structural Integrity 6, 201-207 (2017)

35. V. Kostin, Y. Smorodinskii, Russ J. Nondestruct. Test. 53, 493-504 (2017) 